\title{
Biased Brownian motion in extreme corrugated tubes
}

\author{
S. Martens, ${ }^{1, a)}$ G. Schmid, ${ }^{2}$ L. Schimansky-Geier, ${ }^{1}$ and P. Hänggi ${ }^{2}$ \\ 1) Department of Physics, Humboldt-Universität zu Berlin, Newtonstr. 15, 12489 Berlin, \\ Germany \\ 2) Department of Physics, Universität Augsburg, Universitätsstr. 1, 86135 Augsburg, \\ Germany
}

Biased Brownian motion of point-size particles in a three-dimensional tube with smoothly varying crosssection is investigated. In the fashion of our recent work ${ }^{1}$ we employ an asymptotic analysis to the stationary probability density in a geometric parameter of the tube geometry. We demonstrate that the leading order term is equivalent to the Fick-Jacobs approximation. Expression for the higher order corrections to the probability density are derived. Using this expansion orders we obtain that in the diffusion dominated regime the average particle current equals the zeroth-order Fick-Jacobs result corrected by a factor including the corrugation of the tube geometry. In particular we demonstrate that this estimate is more accurate for extreme corrugated geometries compared to the common applied method using the spatially dependent diffusion coefficient $D(x, f)$. The analytic findings are corroborated with the finite element calculation of a sinusoidal-shaped tube.

PACS numbers: 05.60.Cd, 05.40.Jc, 02.50.Ey, 51.20.+d

Particle transport in micro- and nanostructured channel structures exhibits peculiar characteristics which differs from other transport phenomena occurring for energetic systems. The theoretical modelling involves Fokker-Planck type dynamics in three dimensions which cannot be solved for arbitrary boundary conditions imposed by the geometrical restrictions. Recently, much effort is drawn on a reduction of the complexity of the problem resulting in the so-called FickJacobs approximation in which (infinitely) fast equilibration in certain spatial directions is assumed. Within the present manuscript we derive a reduction method which (i) corresponds in zeroth order in the expansion parameter, which describes the corrugation of the tube wall, to the celebrated Fick-Jacobs result and (ii) extends the validity of the Fick-Jacobs approximation towards extreme corrugated tube structures.

\section{INTRODUCTION}

The transport of large molecules and small particles that are geometrically confined within zeolites ${ }^{2}-\underline{4}$, biological ${ }^{\underline{5}}$ as well as designed nanopores ${ }^{6-8}$, channels or other quasi-one-dimensional systems attracted attention in the last decade. This activity stems from the profitableness for shape and size selective catalysis 9.10 , particle separation and the dynamical characterization of polymers during their translocation $11-15$. In particular, the latter theme which aims at the experimental determination of the structural properties and the amino

a) Electronic mail: steffen.martens@physik.hu-berlin.de acid sequence in DNA or RNA when they pass through narrow openings or the so-called bottlenecks, comprises challenges for technical developments of nanoscaled channel structures ${ }^{14}-17$.

Along with the progress of the experimental techniques the problem of particle transport through corrugated channel structures containing narrow openings and bottlenecks has give rise to recent theoretical activities to study diffusion dynamics occurring in such geometries 18 . Previous studies by Jacobs 19 and Zwanzig 20 ignited a revival of doing research in this topic. The so-called Fick-Jacobs approach ${ }^{18-20}$, accounts for the elimination of transverse stochastic degrees of freedom by assuming a (infinitely) fast equilibration in those transverse directions. The theme found its application for biased particle transport through periodic 3D planar channel structures ${ }^{1,21-25}$ as well as for tubes ${ }^{7,26-28}$ exhibiting smoothly varying side-walls.

Beyond the Fick-Jacobs (FJ) approach, which is suitably applied to channel geometries with smoothly varying side-walls, there exist yet other methods for describing the transport through varying channel structures like cylindrical septate channels ${ }^{29-32}$, tubes formed by spherical compartments ${ }^{33,34}$ or channels containing abrupt changes of cross diameters 35,36 .

In a recent work ${ }^{1}$ we have provided a systematic treatment by using a series expansion of the stationary probability density in terms of a geometric parameter which specifies the channel corrugation for biased particle transport proceeding along a planar three-dimensional channel exhibiting periodically varying, axis symmetric sidewalls. We have demonstrated that the consideration of the higher order corrections to the stationary probability density leads to a substantial improvement of the commonly employed Fick-Jacobs approach towards extreme corrugate channels. The object of this work is to provide an analytic treatment to biased Brownian motion in cylindrical three-dimensional tubes with periodically 


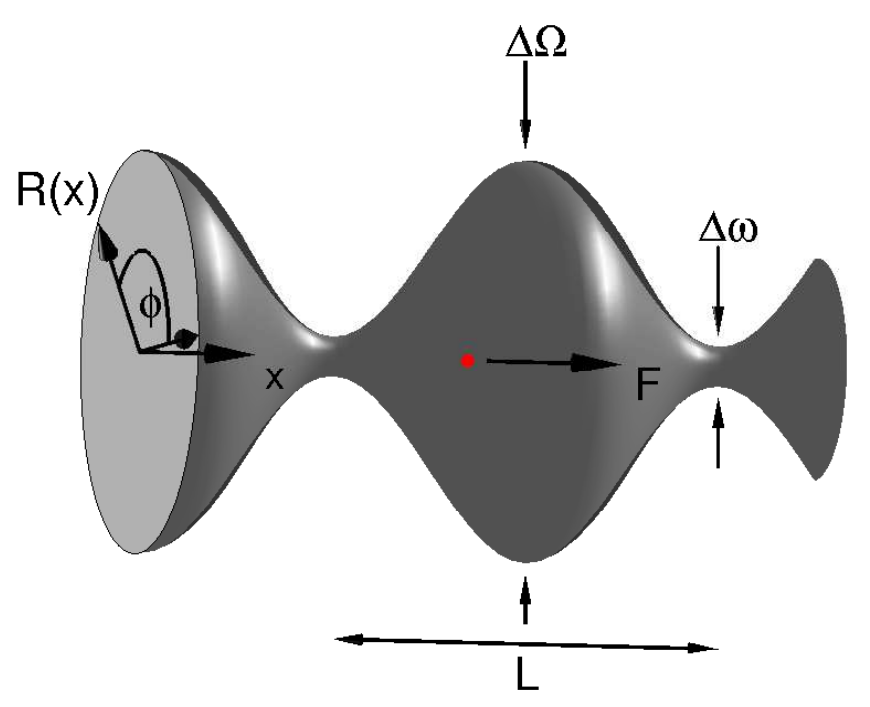

Figure 1. Sketch of a segment of a cylindrical tube with sinusoidally varying radius $R(x)$ that is confining the motion of the overdamped, point-like Brownian particle. The periodicity of the tube structures is $L$, the minimal and maximal tube widths are $\Delta \omega$ and $\Delta \Omega$, respectively. The constant force $F$ pointing in the direction of the tube is applied on the particles.

varying radius.

In Sec. II we introduce the model system: a Brownian particle in a confined tube geometry with periodically modulated boundaries. The central findings, namely the analytic expressions for the probability density are presented in Sec. III] It turns out that the latter scales linearly with the particle velocity derived within the FJ approach, cf. Sec. IV] In Sec. D we calculate the particle mobility and the diffusion coefficient and employ our analytical results to a tube with sinusoidal varying crosssection. Section VI summarizes our findings.

\section{TRANSPORT IN CONFINED STRUCTURES}

The present paper deals with biased transport of overdamped Brownian particles in a cylindrical tube with periodically varying cross-section, respectively, radius $R(x)$. A sketch of a tube segment with period $L$ is shown in Fig. 11 The particles budge within a static fluid with constant friction coefficient $\eta$. As the particle radius (assumed to be point-like) is small compared to the tube radius, hydrodynamic particle-particle interactions as well as hydrodynamic particle-wall interactions can safely be neglected. Further the particles are subjected to an external force with static magnitude $F$ acting along the longitudinal direction of the tube $\mathbf{e}_{x}$, i.e. the corresponding potential is $U(x, r, \phi)=-F x$.

The evolution of the probability density $P(\mathbf{q}, t)$ of finding the particle at the local position $\mathbf{q}=(x, r, \phi)^{T}$ at time $t$ is governed by the three-dimensional Smolu- chowski equation ${ }^{37.38}$, i.e.,

$$
\partial_{t} P(\mathbf{q}, t)+\nabla_{\mathbf{q}} \cdot \mathbf{J}(\mathbf{q}, t)=0,
$$

where

$$
\mathbf{J}(\mathbf{q}, t)=\frac{F}{\eta} P(\mathbf{q}, t) \mathbf{e}_{x}-\frac{k_{B} T}{\eta} \nabla_{\mathbf{q}} P(\mathbf{q}, t)
$$

is the probability current $\mathbf{J}(\mathbf{q}, t)=\left(J^{x}, J^{r}, J^{\phi}\right)^{T}$ associated to the probability density $P(\mathbf{q}, t)$. The Boltzmann constant is $k_{B}$ and $T$ refers to the environmental temperature. At the tube wall the probability current obeys the no-flux boundary condition (bc) caused by the impenetrability of the tube walls, viz. $\mathbf{J}(\mathbf{q}, t) \cdot \mathbf{n}=0$ where $\mathbf{n}$ is the out-pointing normal vector at the tube walls. For a tube with radius $R(x)$ the bc read

$$
R^{\prime}(x) J^{x}(\mathbf{q}, t)=J^{r}(\mathbf{q}, t), \quad \text { for } \quad r=R(x) .
$$

The prime denotes the derivative with respect to $\mathrm{x}$. As a result of symmetry arguments the probability current must be parallel with the tube's centerline at $r=0$

$$
\left.J^{r}(\mathbf{q}, t)\right|_{r=0}=0 .
$$

Further the probability density satisfies the normalization condition $\int_{\text {unit-cell }} P(\mathbf{q}, t) d^{3} \mathbf{q}=1$ as well as the periodicity condition $P(x+m L, r, \phi, t)=$ $P(x, r, \phi, t), \forall m \in \mathbb{Z}$.

Since the external force acts only in longitudinal direction $P(\mathbf{q}, t)$ is radial symmetric. This allows a reduction of the problem's dimensionality from $3 \mathrm{D}$ to $2 \mathrm{D}$ by integrating Eq. (1a) over the angle $\phi$; yielding

$$
\begin{aligned}
\partial_{t} P(x, r, t)= & \frac{k_{B} T}{\eta} \partial_{x}\left[e^{-\frac{U(x, r)}{k_{B} T}} \partial_{x}\left(e^{\frac{U(x, r)}{k_{B} T}} P(x, r, t)\right)\right] \\
& +\frac{k_{B} T}{\eta} \frac{1}{r} \partial_{r}\left[r \partial_{r} P(x, r, t)\right]
\end{aligned}
$$

where the two-point probability density is defined as

$$
P(x, r, t)=\frac{1}{2 \pi} \int_{0}^{2 \pi} d \phi P(x, r, \phi, t) .
$$

Integrating Eq. (3) further over the cross-section and taking the boundary conditions Eqs. (2) into account, one gets

$\partial_{t} P(x, t)=\frac{k_{B} T}{\eta} \partial_{x} \int_{0}^{R(x)} d r r\left[e^{-\frac{U(x, r)}{k_{B} T}} \partial_{x}\left(e^{\frac{U(x, r)}{k_{B} T}} P(x, r, t)\right)\right]$.

Thereby the marginal probability density reads

$$
P(x, t)=\frac{1}{2 \pi} \int_{0}^{R(x)} d r r \int_{0}^{2 \pi} d \phi P(x, r, \phi, t) .
$$


In Ref $\stackrel{1}{\underline{1}}$ we present a perturbation series expansion in terms of a geometric parameter for the problem of biased Brownian dynamics in a planar three-dimensional channel geometry. Below we apply this method for Brownian motion in cylindrical three-dimensional tubes. In doing so we introduce dimensionless variables. We measure the longitudinal length in units of the period length $L$, viz. $\bar{x}=x / L$. For the rescaling of the $r$-coordinate, we introduce the dimensionless aspect parameter $\varepsilon$, i.e. the difference of the widest cross-section of the tube, i.e. $\Delta \Omega$, and the most narrow constriction at the bottleneck, i.e. $\Delta \omega$, in units of the period length, yielding

$$
\varepsilon=\frac{(\Delta \Omega-\Delta \omega)}{L} .
$$

The dimensionless parameter $\varepsilon$ characterizes the deviation of the boundary from the straight tube corresponding to $\varepsilon=0$. Several authors considered different choices for the expansion parameter like the averaged half width $25,39,40$ or the ratio of an imposed anisotropy of the diffusion constants $\varepsilon^{2}=D_{y} / D_{x} 24$.

We next measure, for the case of finite corrugation $\varepsilon \neq 0$, the radius $r$ in units of $\varepsilon L$, i.e. $r=\varepsilon L \bar{r}$ and, likewise, the boundary function $R(x)=\varepsilon L h(x)$. Time is measured in units of $\tau=L^{2} \eta /\left(k_{B} T\right)$ which is twice the time the particle requires to overcome diffusively, at zero bias $F=0$, the distance $L$, i.e. $\bar{t}=t / \tau$. The potential energy is rescaled by the thermal energy $k_{\mathrm{B}} T$, i.e., for the considered situation with a constant force component in longitudinal direction, one gets $\bar{U}:=\bar{U}(x, r)=-F x /\left(k_{\mathrm{B}} T\right)=-f \bar{x}$, with the dimensionless force magnitude 21,23 :

$$
f=\frac{F L}{k_{B} T} .
$$

After scaling the probability distribution reads $p(\overline{\mathbf{q}}, \bar{t})=$ $\varepsilon^{2} L^{3} P(\mathbf{q}, t)$. Below we shall omit the overbar in our notation.

Further we concentrate only on the steady state, i.e., $\lim _{t \rightarrow \infty} p(x, r, t):=p_{\text {st }}(x, r)$, which is in fact, the only state necessary for deriving the key quantities of particle transport like the average particle velocity $\langle\dot{x}\rangle$

$$
\langle\dot{x}\rangle \equiv \lim _{t \rightarrow \infty} \frac{\langle x(t)\rangle}{t}=\int_{0}^{1} d x \int_{0}^{h(x)} d r r J_{\mathrm{st}}^{x}(x, r),
$$

and the effective diffusion coefficient $D_{\text {eff }}$ in force direction. The latter is given by

$$
D_{\mathrm{eff}}=\lim _{t \rightarrow \infty} \frac{\left\langle x^{2}(t)\right\rangle-\langle x(t)\rangle^{2}}{2 t},
$$

and can be calculated by means of the stationary probability density $p_{\text {st }}(x, r)$ using an established method taken from Ref. 11 .
At steady state, the Smoluchowski equation Eq. (3) in dimensionless units becomes

$$
\varepsilon^{2} \partial_{x}\left[e^{-U} \partial_{x}\left(e^{U} p_{\mathrm{st}}(x, r)\right)\right]+\frac{1}{r} \partial_{r}\left[r \partial_{r} p_{\mathrm{st}}(x, r)\right]=0,
$$

and the no-flux boundary conditions Eqs. (2) read

$$
\begin{aligned}
& 0=\left[\partial_{r} p_{\mathrm{st}}(x, r)-\varepsilon^{2} h^{\prime}(x) e^{-U} \partial_{x}\left(e^{U} p_{\mathrm{st}}(x, r)\right)\right]_{r=h(x)}, \\
& 0=\left.\partial_{r} p_{\mathrm{st}}(x, r)\right|_{r=0}
\end{aligned}
$$

\section{ASYMPTOTIC ANALYSIS}

We apply the asymptotic analysis $\frac{1.40}{}$ to the problem stated by Eq. (11) and Eqs. (12). In doing so, we use for the stationary probability density $p_{s t}(x, r)$ (the index $s t$ will be omitted in the following) the ansatz

$$
p(x, r)=\sum_{n=0}^{\infty} \varepsilon^{2 n} p_{n}(x, r)
$$

in the form of a formal perturbation series in even orders of the parameter $\varepsilon$. Substituting these expressions into Eq. (11), we find

$$
\begin{array}{r}
0=\frac{1}{r} \partial_{r}\left[r \partial_{r} p_{0}(x, r)\right]+\sum_{n=1}^{\infty} \varepsilon^{2 n}\left\{\frac{1}{r} \partial_{r}\left[r \partial_{r} p_{n}(x, r)\right]\right. \\
\left.+\partial_{x}\left[e^{-U} \partial_{x}\left(e^{U} p_{n-1}(x, r)\right)\right]\right\}
\end{array}
$$

The no-flux bc at the tube walls $r=h(x)$, cf. Eq. (12a), turns into

$$
\begin{aligned}
0=\partial_{r} p_{0}(x, r)+ & \sum_{n=1}^{\infty} \varepsilon^{2 n}\left\{\partial_{r} p_{n}(x, r)\right. \\
& \left.-h^{\prime}(x) e^{-U} \partial_{x}\left(e^{U} p_{n-1}(x, r)\right)\right\},
\end{aligned}
$$

and the bc at the centerline of the tube $r=0$, cf. Eq. (12b), then reads

$$
0=\sum_{n=0}^{\infty} \varepsilon^{2 n} \partial_{r} p_{n}(x, r)
$$

We claim that the normalization condition for the probability density $p(x, r)$ corresponds to the zeroth solution $p_{0}(x, r)$ that is normalized to unity,

$$
\left\langle p_{0}(x, r)\right\rangle=\int_{0}^{1} d x \int_{0}^{h(x)} d r r p_{0}(x, r)=1 .
$$

Consequently the higher orders in the perturbation series have zero average, $\left\langle p_{n}(x, r)\right\rangle=0, n=1,2,3, \ldots$. 
Further each order $p_{n}$ has to obey the periodic boundary condition $p_{n}(x+m, r)=p_{n}(x, r), \forall m \in \mathbb{Z}$.

InSec. IIIA we demonstrate that the zeroth order of the perturbation series expansion coincides with the Fick-Jacobs equation ${ }^{19,20}$. Referring to $\underline{21.42}$ an expression for the average velocity $\langle\dot{x}\rangle_{0}$ is known. Moreover, in Sec. IIIB, the higher order corrections to the probability density are derived. Using those results we are able to obtain corrections, see in Sec. VB, to the average velocity beyond the zeroth order Fick-Jacobs approximation presented in the next section.

\section{A. Zeroth Order: the Fick-Jacobs equation}

For the zeroth order, Eq. (14) read

$$
0=\frac{1}{r} \partial_{r}\left[r \partial_{r} p_{0}(x, r)\right]
$$

supplemented with the corresponding no-flux boundary condition at $r=0$ as well as at $r=h(x)$

$$
0=\partial_{r} p_{0}(x, r)
$$

We make the ansatz $p_{0}(x, r)=g(x) e^{-U}$ where $g(x)$ is an unknown function which has to be determined from the second order $O\left(\varepsilon^{2}\right)$ balance given by Eq. (14):

$$
0=\partial_{x}\left(e^{-U} g^{\prime}(x)\right)+\frac{1}{r} \partial_{r}\left[r \partial_{r}\left(p_{1}(x, r)\right)\right] .
$$

Integrating the latter over the radius $r$ and taking the no-flux boundary conditions Eqs. (15) into account, one immediately obtains

$$
0=\partial_{x}\left(e^{-A(x)} g^{\prime}(x)\right)
$$

The effective entropic potential $A(x)$ is defined by

$$
e^{-A(x)}=\int_{0}^{h(x)} d r r e^{-U(x, r)},
$$

and for the problem at hand the latter looks explicitly

$$
A(x)=-f x-\ln \left(\frac{h^{2}(x)}{2}\right) .
$$

Note, that upon an irrelevant additive constant, i.e. $\ln (2 \pi)$, the effective entropic potential corresponds to that given in Ref. 19 .

The normalized stationary probability density within the zeroth order reads 37

$$
p_{0}(x, r)=e^{-U} g(x)=\frac{e^{-U(x, r)} \int_{x}^{x+1} e^{A\left(x^{\prime}\right)} d x^{\prime}}{\int_{0}^{1} d x e^{-A(x)} \int_{x}^{x+1} e^{A\left(x^{\prime}\right)} d x^{\prime}},
$$

and, moreover, the marginal probability density Eq. (6) becomes

$$
p_{0}(x)=e^{-A(x)} g(x) .
$$

Expressing next $g(x)$ by $p_{0}(x)$, see Eq. (19), then yields the celebrated stationary Fick-Jacobs equation

$$
0=\partial_{x}\left[e^{-A(x)} \partial_{x}\left(e^{A(x)} p_{0}(x)\right)\right]
$$

derived previously in Ref ${ }^{20,43}$.

Thus, we demonstrate that the leading order term of the asymptotic analysis is equivalent to the FJ-equation. In the FJ equation the problem of biased Brownian dynamics in a confined 3D geometry is replaced by Brownian motion in the tilted periodic one-dimensional potential $A(x)$. In general, the stationary probability density of finding an overdamped Brownian particle budging in a tube with periodically varying cross-section is sufficiently described by Eq. (24) as long as the extension of the bulges of the tube structures is small compared to the periodicity, i.e. $\varepsilon \ll 1$.

Then the average particle current is calculated by integrating the probability flux $J_{0}^{x}$ over the unit-cel1 ${ }^{42,44,45}$

$$
\begin{aligned}
\langle\dot{x}(f)\rangle_{0} & =\int_{0}^{1} d x \int_{0}^{h(x)} d r r J_{0}^{x}(x, r) \\
& =\frac{1-e^{-f}}{\int_{0}^{1} d x e^{A(x)} \int_{x-1}^{x} e^{-A\left(x^{\prime}\right)} d x^{\prime}}
\end{aligned}
$$

In the spirit of linear response theory, the mobility in dimensionless units is defined by the ratio of the mean particle current Eq. (25) and the applied force $f$, yielding

$$
\mu_{0}(f)=\frac{\langle\dot{x}(f)\rangle_{0}}{f} .
$$

Note, that in order to obtain the mobility in physical units one has to multiply $\mu_{0}$ with the mobility of unconfined particles, i.e. $1 / \eta$.

Further, resulting from the normalization condition Eq. (16), the average particle velocity Eq. (9) simplifies to

$$
\langle\dot{x}\rangle=\langle\dot{x}\rangle_{0}-\sum_{n=1}^{\infty} \varepsilon^{2 n}\left\langle\partial_{x} p_{n}(x, r)\right\rangle .
$$

Therefore we derive that the average particle current is composed of (i) the Fick-Jacobs result $\langle\dot{x}\rangle_{0}$, cf. Eq. (25), and (ii) becomes corrected by the sum of the averaged derivatives of the higher orders $p_{n}(x, r)$. We next address the higher order corrections $p_{n}(x, r)$ of the probability density which become necessary for more corrugated structures. 


\section{B. Higher order contributions to the Fick-Jacobs equation}

According to Eq. (14), one needs to iteratively solve

$$
\frac{1}{r} \partial_{r}\left[r \partial_{r} p_{n}(x, r)\right]=\mathfrak{L} p_{n-1}(x, r), \quad n \geq 1,
$$

under consideration of the boundary conditions Eqs. (15). In Eq. (28), we make use of the operator $\mathfrak{L}$, reading $\mathfrak{L}=\left(f \partial_{x}-\partial_{x}^{2}\right)$. Each solution of the second order partial differential equation Eq. (28) possesses two integration constants $d_{n, 1}$ and $d_{n, 2}$. The first one, $d_{n, 1}$, is determined by the no-flux bc at the centerline $r=0$, cf. Eq. (15b), while the second provides the zero average condition $\left\langle p_{n}(x, r)\right\rangle=0, n \geq 1$.

For the first order correction, the determining equation reads

$$
\frac{1}{r} \partial_{r}\left[r \partial_{r} p_{1}(x, r)\right]=2\langle\dot{x}\rangle_{0} \partial_{x}\left(\frac{1}{h^{2}(x)}\right),
$$

and after integrating twice over $r$, we obtain

$$
p_{1}(x, r)=-\langle\dot{x}\rangle_{0}\left(\frac{h^{\prime}(x)}{h^{3}(x)}\right) r^{2} .
$$

Hereby, as requested above, the first integration constant $d_{1,1}(x)$ is set to 0 in order to fulfill the no-flux bcs, and the second must provide the normalization condition Eq. (16), i.e. $d_{1,2}=0$. One notices that the first correction to the probability density becomes positive if the confinement is constricting, i.e. for $h^{\prime}(x)<0$ and $\langle\dot{x}\rangle_{0} \neq 0$. In contrast, the probability density becomes less in unbolting regions of the confinement, i.e. for $h^{\prime}(x)>0$. Please note, that the first order correction scales linearly with the average particle current $\langle\dot{x}\rangle_{0}$. Overall, the break of spatial symmetry observed within numerical simulations in previous works ${ }^{22,46}$ is reproduced by this very first order correction. Particularly, with increasing forcing, the probability for finding a particle close to the constricting part of the confinement increases, cf. Ref. 22,46 .

Upon recursively solving, the higher order corrections, $n \geq 1$, are given by

$$
\begin{aligned}
p_{n}(x, r) & =\mathfrak{L}^{n} p_{0}(x, r) \frac{r^{2 n}}{\left(2^{n} n !\right)^{2}}+d_{n, 2} \\
& =2\langle\dot{x}\rangle_{0} \frac{r^{2 n}}{\left(2^{n} n !\right)^{2}} \mathfrak{L}^{n-1} \partial_{x}\left(\frac{1}{h^{2}(x)}\right)+d_{n, 2}
\end{aligned}
$$

where the operator $\mathfrak{L}$ applied $n$-times yields the expression

$$
\mathfrak{L}^{n}=\sum_{k=0}^{n}\left(\begin{array}{l}
n \\
k
\end{array}\right)(-1)^{k} f^{n-k} \frac{\partial^{n+k}}{\partial x^{n+k}} .
$$

Note that each single order $p_{n}(x, r)$, cf. Eq. (31), satisfies the normalization condition, the bc at the centerline but does not obey the bc at the tube wall, see Eq. (15a). The stationary probability density $p(x, r)$ is obtained by summing all correction terms, cf. Eq. (13), yielding

$p(x, r)=p_{0}(x, r)+\sum_{n=1}^{\infty} \varepsilon^{2 n}\left(\mathfrak{L}^{n} p_{0}(x, r) \frac{r^{2 n}}{\left(2^{n} n !\right)^{2}}+d_{n, 2}\right)$.

Inserting Eq. 33 into the equation for the no-flux bc at the tube wall, cf. Eq. (12a), results to

$$
\begin{aligned}
0 & \equiv \sum_{n=0}^{\infty} \varepsilon^{2 n+2} \partial_{x} \int_{0}^{h(x)} d r r e^{-U(x, r)} \partial_{x}\left(e^{U(x, r)} p_{n}(x, r)\right) \\
& =\varepsilon^{2} \partial_{x} \int_{0}^{h(x)} d r r e^{-U(x, r)} \partial_{x}\left(e^{U(x, r} p(x, r)\right) .
\end{aligned}
$$

According to Eq. (5) the latter equals zero in the steady state.

Summing up, the exact solution for the stationary probability density of finding a biased Brownian particle in tube is given by Eq. (33). The latter solves the corresponding Smoluchowski equation Eq. (11) under satisfaction of the normalization as well as the periodicity requirements. More importantly the solution, cf. Eq. (33), obeys the no-flux boundary conditions at the centerline $r=0$ as well as at the tube wall $r=h(x)$. Further one notices that $p(x, r)$ is fully determined by the Fick-Jacobs results $p_{0}(x, r)$. Caused by $\mathfrak{L} p_{0}(x, r) \propto\langle\dot{x}(f)\rangle_{0}$, the contribution of the higher order corrections to the $2 \mathrm{D}$ probability density scales linear with the average particle current in the FJ limit $\langle\dot{x}\rangle_{0}$, cf. Eq. (31). The latter is determined by the break of spatial symmetry induced by the external force. Consequently, in the absence of the external force $f=0$ the stationary probability density equals the zeroth order contribution $p(x, r)=p_{0}(x, r)=$ const despite the value of $\varepsilon$.

According to Eq. (27), one recognizes that the average particle current scales with the average particle current obtained from the Fick-Jacobs formalism $\langle\dot{x}\rangle_{0}$ for all values of $\varepsilon$. Therefore, in order to validate the obtained results for $p(x, r)$ as well as to derive correction to the mean particle current it is required to calculate $\langle\dot{x}\rangle_{0}$ first.

\section{TRANSPORT QUANTITIES FOR A SINUSOIDALLY SHAPED TUBE}

In the following we study the key transport quantities like the particle mobility $\mu(f)$ and the effective diffusion coefficient $D_{\text {eff }}(f)$ of point-like Brownian particles moving in a sinusoidally-shaped ${ }^{23}$ tube. The dimensionless boundary function $h(x)$ reads

$$
h(x)=\frac{1}{4}\left(\frac{1+\delta}{1-\delta}+\sin (2 \pi x)\right),
$$


and is illustrated in Fig. 1. The function $h(x)$ is solely governed by the aspect ratio of the minimal and maximum tube width $\delta=\Delta \omega / \Delta \Omega$. Obviously different realizations of tube geometries can possess the same value of $\delta$. The number of orders have to taken into account in the perturbation series Eq. (13), respectively, the applicability of the Fick-Jacobs approach to the problem, depends only on the value of the geometric parameter $\varepsilon=\Delta \Omega(1-\delta) / L$ for a given aspect ratio $\delta$.

First, we obtain the particle mobility $\mu_{0}$ within the zeroth-order (Fick-Jacobs approximation). Referring to Eqs. (25) and (26) the dimensionless mobility is given by

$$
\mu_{0}(f)=\frac{1-e^{-f}}{f \int_{0}^{1} d x e^{-f x^{\prime}} / h^{2}\left(x^{\prime}\right) \int_{x-1}^{x} e^{f x} h^{2}(x) d x^{\prime}}
$$

For the considered sinusoidal boundary function, cf. Eq. (35), we obtain

$$
\begin{aligned}
\frac{1}{\mu_{0}(f)}= & \frac{1}{2{\sqrt{b^{2}-1}}^{3}}\left\{b\left(2 b^{2}+1\right)-\frac{4 b f^{2}}{f^{2}+(2 \pi)^{2}}\right. \\
& \left.+\frac{\left(2{\sqrt{b^{2}-1}}^{3}-2 b^{3}+3 b\right) f^{2}}{f^{2}+(4 \pi)^{2}}\right\},
\end{aligned}
$$

with the substitute $b=(1+\delta) /(1-\delta)$. Caused by the reflection symmetry of the boundary function $h(x)$ the particle mobility obeys $\mu_{0}(-f)=\mu_{0}(f)$. Thus it is sufficient to discuss only the behavior for $f \geq 0$.

In the limiting case of infinite large force strength, the mobility goes to

$$
\lim _{f \rightarrow \infty} \mu_{0}(f)=1
$$

With decreasing force magnitude $f$ the mobility decreases as well till $\mu_{0}(f)$ attains the asymptotic value

$$
\lim _{f \rightarrow 0} \mu_{0}(f)=\frac{2 \sqrt{\delta}}{1+\delta} \frac{8 \delta}{3 \delta^{2}+2 \delta+3} .
$$

In the diffusion dominated regime, $|f| \ll 1$, the Sutherland-Einstein relation emerges 47,48 and thus the dimensionless mobility equals the dimensionless effective diffusion coefficient:

$$
\lim _{f \rightarrow 0} \mu(f)=\lim _{f \rightarrow 0} D_{\text {eff }}(f) .
$$

In the limit of vanishing bottleneck width, i.e. $\delta \rightarrow 0$, the mobility, respectively, $D_{\text {eff }}$ tends to 0 . In contrast, for straight tubes corresponding to $\delta=1$, i.e. $\varepsilon=0$, the mobility as well as the effective diffusion coefficient equal their free values which are one in the considered scaling.

In a recent work ${ }^{1}$, we studied the biased Brownian motion in a planar three-dimensional channel geometry with periodically varying width $2 h(x)$. We found that the asymptotic value for the mobility is given by $2 \sqrt{\delta} /(1+\delta)$

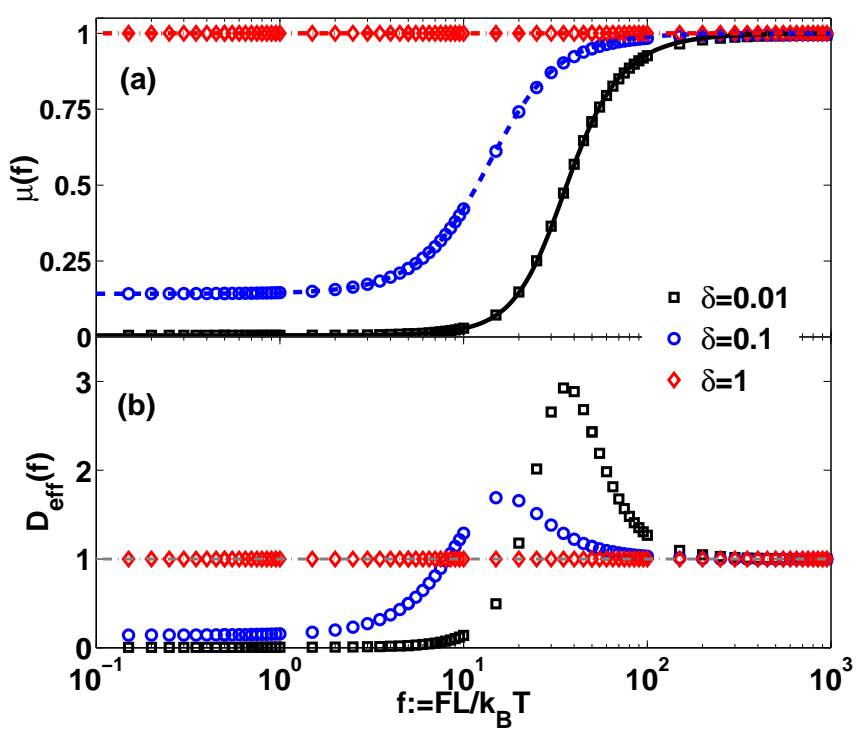

Figure 2. (Color online) The particle mobility (a) and the effective diffusion constant (b) for a Brownian particle moving inside a sinusoidal tube are depicted as function of the external force magnitude $f$. The maximum tube width is kept fixed, viz. $\Delta \Omega=0.1$, while the aspect ratio is varied $\delta=0.01,0.1,1$, respectively, the corresponding values for $\varepsilon$ are $\varepsilon=0.099,0.09,0$. The symbols correspond to the numerical obtained mobility, respectively, the effective diffusion coefficient. In subfigure (a) the lines correspond to the analytic result, cf. Eq. (37).

for $f \rightarrow 0$, cf. Eq. (45) in Ref 1 . Comparing this result with the asymptotic value Eq. (39) one notices that the mobility and the effective diffusion coefficient in a tube, respectively, are less compared to case of a planar channel geometry.

In Fig. 2 we depict the dependence of the mobility $\mu(f)$ and the effective diffusion coefficient $D_{\text {eff }}(f)$ on the external force magnitude $f$. The numerical results are obtained by solving the stationary Smoluchowski equation Eq. (1a) using finite element method 49 and subsequently calculating the average particle current according to Eq. (9). In order to determine the effective diffusion coefficient $D_{\text {eff }}(f)$, one has to solve numerically the reaction-diffusion equation for the $B$-field 39,41 .

Referring to Fig. 2(a) one notices that the analytic predictions for the particle mobility Eq. (37) are corroborated by numerics. Further, one observes that for the case of smoothly varying tube geometry, i.e. $\Delta \Omega / L \ll 1$, the analytic result is in excellent agreement with the numerics for a large range of dimensionless force magnitudes $f$, indicating the applicability of the Fick-Jacobs approach. As long as the extension of the bulges of the tube structures is small compared to the periodicity, sufficiently fast transversal equilibration, which serves as fundamental ingredient for the validity of the Fick-Jacobs approximation is taking place.

The effective diffusion coefficient $D_{\text {eff }}(f)$ exhibits a non-monotonic dependence versus the dimensionless 


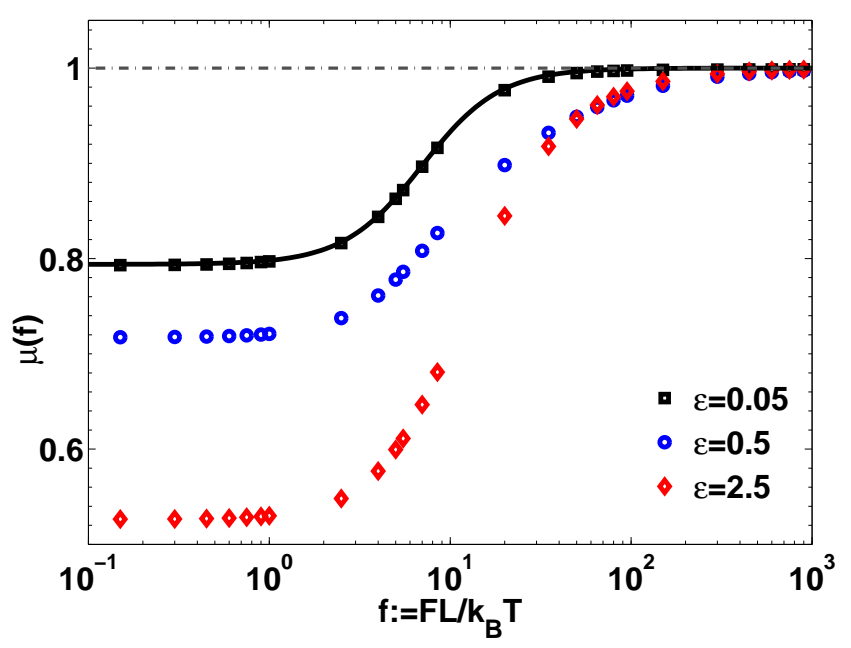

Figure 3. (Color online) The influence of the geometric parameter $\varepsilon$ on the particle mobility is presented. The value of $\varepsilon$ is varied, viz. $\varepsilon=0.05,0.5,2.5$, while the aspect ratio is kept fixed, viz. $\delta=0.5$. The solid line corresponds to the analytic result Eq. (37) while the dash-dotted line indicates the asymptotic value one.

force $f$, see Fig. 2(b). It starts out with a value that is less than the free diffusion constant in the diffusion dominated regime,i.e. $|f| \ll 1$. According to the SutherlandEinstein-relation the value equals the mobility value, cf. Eq. (39). Then it reaches a maximum with increasing $f$ and finally approaches the value of the free diffusion from above. Further one notices that the location of the diffusion peak as well as the peak height depends on the aspect ratio $\delta$. With decreasing width at the bottleneck, while keeping the maximum width $\Delta \Omega$ fix, the diffusion peak is shifted towards larger force magnitude $f$. Simultaneously the peak height grows. In the limit of a straight tube, i.e. $\delta \rightarrow 1$, as expected the effective diffusion coefficient coincide with its free value which is one in the considered scaling.

In Fig. 3 we present the impact of the expansion parameter $\varepsilon$ on the particle mobility. It turns out that for values of $\varepsilon \lesssim 0.1$ the Fick-Jacobs approximation is in very good agreement with the simulation. With increasing geometric parameter $\varepsilon$ the difference between the FJ-result (solid line) and the numerics is growing. The more available space in the tube leads to a decrease of the particle mobility, respectively, of the effective diffusion coefficient. Consequently, the higher order corrections to the stationary probability density $p(x, r)$, see Eq. (13), respectively, the corrections to the mobility, cf. Eq. (27), need to be included in order to provide a better agreement.

\section{CORRECTIONS TO THE MOBILITY AND DIFFUSION COEFFICIENT}

A common used way to include the corrugation of the channel structure bases on the concept of the spatially dependent diffusion coefficient $D(x, f)$ which was introduced by Zwanzig 20 and subsequently supported by the study of Reguera and Rubi $\frac{43}{}$. Zwanzig obtained the FJ equation, cf. Eq. (24), from the full 3D Smoluchowski equation upon eliminating the transverse degrees of freedom supposing infinitely fast relaxation. In a more detailed view, we have to notice that diffusing particles can flow out from/ or towards the wall in $r$-direction only at finite time. These finite relaxation processes are included by scaling the diffusion constant in longitudinal direction by a position dependent function, viz. $D(x, f)$. The latter substitutes the constant diffusion coefficient which is one in the considered scaling - in the common stationary FJ equation, cf. Eq. (24), yielding

$$
0=\partial_{x}\left[D(x, f) e^{-A(x)} \partial_{x}\left(e^{A(x)} p(x)\right)\right] .
$$

According to Eq. (41) an expression for the particle mobility that is similar to the Stratonovich formula 21.42 for the mobility in titled periodic energy landscapes, but now including the the spatial diffusion coefficient $D(x, f)$, can be derived ${ }^{21,22}$. In the diffusion dominated regime, i.e. $|f| \ll 1$, this expression simplifies to the Lifson-Jackson formula 22,50

$$
\lim _{f \rightarrow 0} \mu(f)=\lim _{f \rightarrow 0} D_{\text {eff }}(f)=\frac{1}{\left\langle h^{2}(x)\right\rangle\left\langle\frac{1}{D(x, 0) h^{2}(x)}\right\rangle},
$$

with the period average $\langle\cdot\rangle=\int_{0}^{1} \cdot d x$. Unfortunately, for many boundary functions $h(x)$ it is impossible to analytically evaluate the expression Eq. (42).

\section{A. Spatially dependent diffusion coefficient $D(x, f)$}

A first systematically treatment taking the finite diffusion time into account was presented by Kalinay and Percus (KP) $)^{24,51}$. Their suggested mapping procedure enables the derivation of higher order corrections in terms of an expansion parameter $\varepsilon_{K P}^{2}$, which is the ratio of the diffusion constants in the longitudinal and transverse directions. Within this scaling, KP have shown that the fast transverse modes (transients) separate from the slow longitudinal ones and therefore the transients can be projected out by integration over the transverse directions.

In what follows we present a derivation for the spatially dependent diffusion coefficient $D(x, f)$ which based on our previously considered perturbation series expansion for the stationary probability density Sec. III According to Eq. (5) the marginal probability current $J^{x}(x)$, equivalent to Eq. (41), can be derived in an alternative way using the stationary two-point probability density 
$p(x, r)$, yielding

$$
\begin{aligned}
-J^{x}(x) & =D(x, f) e^{-A(x)} \partial_{x}\left(e^{A(x)} p(x)\right) \\
& =\int_{0}^{h(x)} d r r e^{-U(x, r)} \partial_{x}\left(e^{U(x, r)} p(x, r)\right) .
\end{aligned}
$$

The second equality determines the sought-after spatial dependent diffusion coefficient $D(x, f)$.

One immediately notices that the relation Eq. (43) simplifies to $D(x, f) f p(x)=\int_{0}^{h(x)} d r r f p(x, r)$ in the force dominated regime $|f| \gg 1$. Then it follows that the spatially diffusion coefficient equals the free one, which is one in the considered scaling,

$$
\lim _{f \rightarrow \infty} D(x, f)=1 \text {. }
$$

In the opposite limit of small force strengths, i.e. for $|f| \ll 1$, diffusion is the dominating process. Then Eq. (43) simplifies to

$$
D(x, f) h^{2}(x) \partial_{x}\left(\frac{p(x)}{h^{2}(x)}\right)=\int_{0}^{h(x)} d r r \partial_{x} p(x, r) .
$$

Inserting our result for the stationary probability density $p(x, r)$, cf. Eq. (33), into Eq. (45) we determine an expression for $D(x, f)$. In compliance with Ref ${ }^{24}$, we make the ansatz that all but the first derivative of the boundary function $h(x)$ are negligible. Then the $n$-times applied operator $\mathfrak{L}$, cf. Eq. (32), simplifies to $\mathfrak{L}^{n}=(-1)^{n} \frac{\partial^{2 n}}{\partial x^{2 n}}$, yielding,

$p_{n}(x, r)=2(-1)^{n}\langle\dot{x}\rangle_{0} \frac{(2 n) !}{\left(2^{n} n !\right)^{2}} \frac{\left(h^{\prime}\right)^{2 n-1}}{h^{2 n+1}} r^{2 n}+O\left(h^{\prime \prime}(x)\right)$.

Inserting the latter into Eq. (43) and calculating the complete sum, one finds

$$
\lim _{f \rightarrow 0} D(x, f)=\frac{1}{\sqrt{1+\left(\varepsilon h^{\prime}(x)\right)^{2}}}+O\left(h^{\prime \prime}(x)\right)
$$

for the spatially dependent diffusion coefficient $D(x, f)$ in the diffusion dominated regime, i.e. for $|f| \ll 1$. Using our above presented series expansion for the stationary probability density $p(x, r)$ we confirm the expression for the spatially dependent diffusion coefficient $D(x, f)$ previously derived by Reguera and Rubi ${ }^{43}$ and KP24,51.

\section{B. Corrections based on perturbation series expansion}

Next, we derive an estimate for the mean particle current $\langle\dot{x}(f)\rangle$ based on the higher expansion orders $p_{n}(x, r)$. Referring to Eq. (27), the average particle current is composed of (i) the Fick-Jacobs result $\langle\dot{x}\rangle_{0}$, cf. Eq. (25), and (ii) becomes corrected by the sum of the averaged derivatives of the higher orders $p_{n}(x, r)$. Immediately one notices that the integration constant $d_{n, 2}$, resulting from the normalization condition Eq. (16), does not influence the result for the average particle velocity, cf. Eq. (27).

We concentrate on the diffusion dominated limit $|f| \ll 1$ and further we make the ansatz that all but the first derivative of the boundary function $h(x)$ are negligible ${ }^{1,24}$. Then the partial derivative of $p_{n}(x, r)$ with respect to $x$ simplifies to

$\partial_{x} p_{n}(x, r)=2\langle\dot{x}\rangle_{0}(-1)^{n+1} \frac{\left(h^{\prime}\right)^{2 n}}{h^{2 n+2}} \frac{(2 n+1) ! r^{2 n}}{\left(2^{n} n !\right)^{2}}+O\left(h^{\prime \prime}(x)\right)$.

Inserting the latter into Eq. (27) and integrating the results over one unit-cell, results in

$$
\begin{aligned}
& \lim _{f \rightarrow 0}\langle\dot{x}(f)\rangle \simeq \lim _{f \rightarrow 0}\langle\dot{x}(f)\rangle_{0} \sum_{n=0}^{\infty} \frac{(-1)^{n}(2 n+1) !}{2^{2 n+1}(n !)(n+1) !}\left\langle\left(\varepsilon h^{\prime}(x)\right)^{2 n}\right\rangle \\
& \simeq \lim _{f \rightarrow 0}\langle\dot{x}(f)\rangle_{0}\left\langle\frac{2}{\left(\varepsilon h^{\prime}(x)\right)^{2}}\left(1-\frac{1}{\sqrt{1+\left(\varepsilon h^{\prime}(x)\right)^{2}}}\right)\right\rangle .
\end{aligned}
$$

We obtain that the average transport velocity is obtained as the product of the zeroth-order Fick-Jacobs result and the expectation value of a complicated function including the slope of the boundary for $|f| \ll 1$. In the previously studied case of biased Brownian motion in a $3 \mathrm{D}$ planar channel geometry ${ }^{\underline{1}}$ we have found that the average transport velocity is obtained as the product of the zeroth-order Fick-Jacobs result and the expectation value of the spatially dependent diffusion coefficient $\langle D(x, 0)\rangle$. In contrast to the 3D planar geometry, for biased transport in extreme corrugated tubes the corrections term to the particle velocity does not coincide with the expectation value of $D(x, 0)$, see Eq. (47).

Calculating the expectation value in Eq. (49) for the considered tube geometry, cf. Eq. (35), yields to the estimate

$$
\lim _{f \rightarrow 0}\langle\dot{x}(f)\rangle \simeq \lim _{f \rightarrow 0}\langle\dot{x}(f)\rangle_{0}{ }_{2} F_{1}\left(\frac{1}{2}, \frac{1}{2}, 2,-\left(\frac{\varepsilon \pi}{2}\right)^{2}\right),
$$

where ${ }_{2} F_{1}(\cdot)$ is the first hypergeometric function. We derive that in the diffusion dominated regime the average velocity is obtained as the product of the zeroth-order Fick-Jacobs result and the correction term ${ }_{2} F_{1}(\cdot)$ including the corrugation of the tube structure. Referring to the Sutherland-Einstein relation, cf. Eq. (40), if the average current $\langle\dot{x}(f)\rangle_{0}$ (or the effective diffusion coefficient $\left.D_{\text {eff }}^{0}(f)\right)$ is known in the zeroth order, the higher order corrections to both quantities can be obtained according to Eq. (50).

We have to emphasize that considering only the first derivative of the boundary function $h^{\prime}(x)$ 


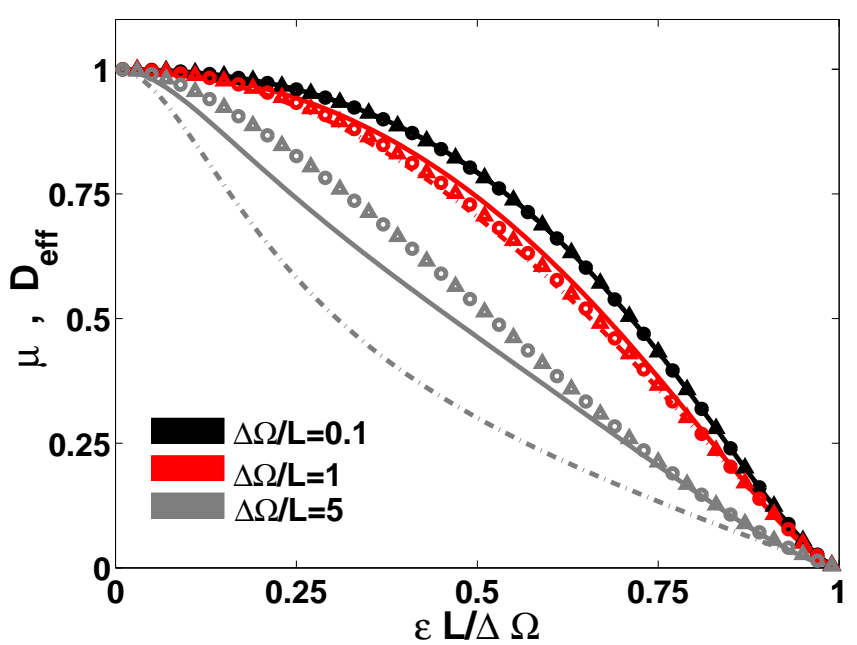

Figure 4. (Color online) Comparison of the analytic theory versus precise numerics (in dimensionless units): The mobility and the effective diffusion constant for a Brownian particle moving inside a tube with sinusoidal varying radius are depicted as function of geometric parameter $\varepsilon$ in units of the maximum channel width $\Delta \Omega$. The latter is varied $\Delta \Omega / L=0.1,1,5$ (from top to bottom) while the external bias is kept fixed $f=10^{-3}$ (corresponding to the diffusion dominated regime). The symbols correspond to the numerical obtained mobility (triangles) and the effective diffusion coefficient (circles). The solid lines correspond to analytic higher order result, cf. Eq. (50). The zeroth order - Fick-Jacobs results given by Eq. (39) collapse to a single curve hidden by the solid line for $\Delta \Omega / L=0.1$. In addition the numerical evaluation of Eq. (42) is represented by the dash-dotted lines.

results in an additional term proportional to $\varepsilon_{2}^{2} F_{1}\left(3 / 2,3 / 2,3,-(\varepsilon \pi / 2)^{2}\right)$. Taking further the second derivative $h^{\prime \prime}(x)$ into accounts indicates that this second term is negligible compared to ${ }_{2} F_{1}(1 / 2, \ldots)$ for arbitrarily value of $\varepsilon$.

In Fig. 4, we present the dependence of the $\mu(f)$ (triangles) and $D_{\text {eff }}(f)$ (circles) on the slope parameter $\varepsilon$ for $f=10^{-3}$. One observes that the numerical results for the effective diffusion coefficient $D_{\text {eff }}(f)$ and the mobility $\mu(f)$ coincide for all values of $\varepsilon$, thus corroborating the Sutherland-Einstein relation. In addition, the FickJacobs result, given by Eq. (39), the higher order result (solid lines), see Eq. (50), and the numerical evaluation of the Lifson-Jackson formula using $D(x, 0)$ (dash-dotted lines), cf. Eq. (42), are depicted in Fig. 4.

For the case of smoothly varying tube geometry, i.e. $\Delta \Omega / L \ll 1$, all analytic expressions are in excellent agreement with the numerics, indicating the applicability of the Fick-Jacobs approach. In virtue of Eq. (7), the geometric parameter is defined by $\varepsilon=(\Delta \Omega-\Delta \omega) / L$ and hence the maximal value of $\varepsilon$ equals $\Delta \Omega / L$. Consequently the influence of the higher expansion orders $\varepsilon^{2 n}\left\langle\partial_{x} p_{n}(x, r)\right\rangle$ on the average velocity Eq. (27) and on the mobility, respectively, becomes negligible if the max- imum tube's width $\Delta \Omega / L$ is small.

With increasing maximum width the difference between the FJ-result Eq. (39) and the numerics is growing. Specifically, the FJ-approximation overestimates the mobility $\mu$ and the effective diffusion coefficient $D_{\text {eff }}$. Consequently the corrugation of the tube geometry needs to be included. The consideration of $D(x, 0)$, cf. Eq. (42) provides a good agreement for a wide range of $\varepsilon$-values as long as the maximum width $\Delta \Omega / L$ is on the scale to the period length of the tube, i.e. $\Delta \Omega / L \sim 1$. Upon further increasing the maximum width $\Delta \Omega / L$ diminishes the range of applicability of the presented concept. In detail the expression Eq. (42) drastically underestimate the numerical results due to the neglect of the higher derivatives of the boundary function $h(x)$. Put differently, the higher derivatives of $h(x)$ become significant for $\Delta \Omega / L \gtrsim 1$.

In contrast, one notices that the result for the mobility and the diffusion coefficient, respectively, based on the higher order corrections to the stationary probability density Eq. (50) is in very good agreement with the numerics. For tube geometries where the maximum width $\Delta \Omega / L$ is on the scale to the period length, i.e. $\Delta \Omega / L \sim 1$, the correction estimate matches perfectly with the numerical results. Further increase of the tube width results in a small deviation from the simulation results.

\section{SUMMARY AND CONCLUSION}

In summary, we have considered the transport of pointsized Brownian particles under the action of a constant and uniform force field through a 3D tube. The crosssection, respectively, the radius of the tube varies periodically.

We have presented a systematic treatment of particle transport by using a perturbation series expansion of the stationary probability density in terms of a smallness parameter which specifies the corrugation of the tube walls. In particular, it turns out that the leading order term of the series expansion is equivalent to the well-established Fick-Jacobs approach 19,20. The higher order corrections to the probability density become significant for extreme bending of the tube's side-walls. Analytic results for each order of the perturbation series have been derived. Similar to biased Brownian motion in a 3D planar channel all higher order corrections to the stationary probability density scale with the average particle current obtained from the Fick-Jacobs formalism.

Moreover, for the diffusion dominated regime, i.e. for small forcing $|f| \ll 1$, we calculate the correction to the mean particle velocity originated by the tube's corrugation using the series expansion for the stationary probability density. According to the SutherlandEinstein relation, the obtained relation is also valid for the effective diffusion coefficient. In addition, by using the higher order corrections we present an alternative derivation for the spatially dependent diffusion coefficient 
$D(x, f)$ which substitutes the constant diffusion coefficient present in the common Fick-Jacobs equation based on similar assumptions as those suggested by Kalinay and Percus as well as by Rubi and Reguera.

Finally, we have applied our analytic results to a specific example, namely, the particle transport through a tube with sinusoidally varying radius $R(x)$. We corroborate our theoretical predictions for the mobility and the effective diffusion coefficient with precise numerical results of a finite element calculation of the stationary Smoluchowski-equation.

In conclusion, the consideration of the higher order corrections leads to a substantial improvement of the Fick-Jacobs-approach, which corresponds to the zeroth order in our perturbation analysis, towards more winding boundaries of the tube. Notably, we have shown that the common approach using the the spatially dependent diffusion coefficient $D(x, f)$ fails for extreme corrugated tube geometries.

\section{ACKNOWLEDGMENTS}

This article is dedicated to the memory of Dr. Frank Moss. Frank was an outstanding and enthusiastic scientist who was a true pioneer in the field of stochastic resonance $\frac{52,53}{3}$ and in the application of stochastic nonlinear dynamics to biological systems $54-56$. We will always remember him.

This work has been supported by the VW Foundation via project I/83903 (L.S.-G., S.M.) and I/83902 (P.H., G.S.). P.H. acknowledges the support the excellence cluster "Nanosystems Initiative Munich" (NIM).

\section{REFERENCES}

${ }^{1}$ S. Martens, G. Schmid, L. Schimansky-Geier, and P. Hänggi, Phys. Rev. E 83, 051135 (2011)

${ }^{2}$ F. Keil, R. Krishna, and M. Coppens, Rev. Chem. Eng. 16, 71 (2000).

${ }^{3}$ E. Beerdsen, D. Dubbeldam, Phys. Rev. Lett. 95, 164505 (2005). ${ }^{4}$ E. Beerdsen, D. Dubbeldam, and B. Smit, Phys. Rev. Lett. 96, 044501 (2006).

${ }^{5}$ B. Hille, Ion Channels of Excitable Membranes, 3rd ed. (Sinauer Associates, 2001).

${ }^{6}$ C. Kettner, P. Reimann, P. Hänggi, and F. Müller, Phys. Rev. E 61, 312 (2000).

${ }^{7}$ A. M. Berezhkovskii, M. A. Pustovoit, and S. M. Bezrukov, J. Chem. Phys. 126, 134706 (2007).

${ }^{8}$ D. Pedone, M. Langecker, A. M. Muenzer, R. Wei, R. D. Nagel, and U. Rant, J. Phys. Condens. Matter 22, 454115 (2010).

${ }^{9}$ K.-L. Cheng, Y.-J. Sheng, and H.-K. Tsao, J. Chem. Phys. 129, 184901 (2008)

${ }^{10}$ W. Riefler, G. Schmid, P. S. Burada, and P. Hänggi, J. Phys. Condens. Matter 22, 454109 (2010).

${ }^{11}$ M. Muthukumar, Phys. Rev. Lett. 86, 3188 (2001)

${ }^{12}$ S. Matysiak, A. Montesi, M. Pasquali, A. B. Kolomeisky, and C. Clementi, Phys. Rev. Lett. 96, 118103 (2006)

${ }^{13}$ A. Cacciuto and E. Luijten, Phys. Rev. Lett. 96, 238104 (2006)
${ }^{14}$ C. Dekker, Nature Nanotech. 2, 209 (2007).

${ }^{15}$ P. Hänggi and F. Marchesoni, Rev. Mod. Phys. 81, 387 (2009)

${ }^{16}$ U. Keyser, B. Koeleman, S. V. Dorp, D. Krapf, R. Smeets, S. Lemay, N. Dekker, and C. Dekker, Nature Physics 2, 473 (2006).

${ }^{17}$ S. Howorka and Z. Siwy, Chem. Soc. Rev. 38, 2360 (2009).

${ }^{18}$ P. S. Burada, P. Hänggi, F. Marchesoni, G. Schmid, and P. Talkner, ChemPhysChem 10, 45 (2009).

${ }^{19}$ M. Jacobs, Diffusion Processes (Springer, New York, 1967).

${ }^{20}$ R. Zwanzig, J. Chem. Phys., 3926 (1992).

${ }^{21}$ D. Reguera, G. Schmid, P. S. Burada, J. M. Rubi, P. Reimann, and P. Hänggi, Phys. Rev. Lett. 96, 130603 (2006).

${ }^{22}$ P. S. Burada, G. Schmid, D. Reguera, J. M. Rubi, and P. Hänggi, Phys. Rev. E 75, 051111 (2007).

${ }^{23}$ P. S. Burada, G. Schmid, P. Talkner, P. Hänggi, D. Reguera, and J. M. Rubi, BioSystems 93, 16 (2008).

${ }^{24}$ P. Kalinay and J. K. Percus, Phys. Rev. E 74, 041203 (2006).

${ }^{25}$ X. Wang and G. Drazer, Phys. Fluid 22, 122004 (2010)

${ }^{26}$ Bao-quan $\mathrm{Ai}$ and Liang-gang Liu, Phys. Rev. E 74, 051114 (2006)

${ }^{27}$ L. Dagdug, M.-V. Vazquez, A. M. Berezhkovskii, and S. M. Bezrukov, J. Chem. Phys. 133, 034707 (2010)

${ }^{28}$ L. Dagdug, A. M. Berezhkovskii, Y. A. Makhnovskii, V. Y. Zitserman, and S. M. Bezrukov, J. Chem. Phys. 134, 101102 (2011)

${ }^{29}$ F. Marchesoni, J. Chem. Phys. 132, 166101 (2010).

${ }^{30}$ M. Borromeo and F. Marchesoni, Chem. Phys. 375, 536 (2010).

${ }^{31}$ P. Hänggi, F. Marchesoni, S. Savel'ev, and G. Schmid, Phys. Rev. E 82, 041121 (2010)

${ }^{32}$ Y. A. Makhnovskii, A. M. Berezhkovskii, L. V. Bogachev, and V. Y. Zitserman, J. Phys. Chem. B 115, 3992 (2011)

${ }^{33}$ A. M. Berezhkovskii and L. Dagdug, J. Chem. Phys. 133, 134102 (2010).

${ }^{34}$ A. M. Berezhkovskii, L. Dagdug, Y. A. Makhnovskii, and V. Y. Zitserman, J. Chem. Phys. 132, 221104 (2010).

${ }^{35}$ P. Kalinay and J. K. Percus, Phys. Rev. E 82, 031143 (2010)

${ }^{36}$ Y. Makhnovskii, A. M. Berezhkovskii, and V. Y. Zitserman, ChemiPhys 370, 238 (2010)

${ }^{37}$ H. Risken, The Fokker-Planck Equation, 2nd ed. (Springer, Berlin, 1989).

${ }^{38}$ P. Hänggi and H. Thomas, Phys. Rep. 88, 207 (1982)

${ }^{39}$ N. Laachi, M. Kenward, E. Yariv, and K. Dorfman, EPL 80, 50009 (2007).

${ }^{40}$ E. Yariv and K. D. Dorfman, Phys. Fluids 19, 037101 (2007).

${ }^{41} \mathrm{H}$. Brenner and D. A. Edwards, Macrotransport Processes (Butterworth-Heinemann, Boston, 1993).

${ }^{42}$ R. L. Stratonovich, Radiotekh. Elektron. (Moscow) 3, 497 (1958).

${ }^{43}$ D. Reguera and J. M. Rubi, Phys. Rev. E 64, 061106 (2001).

${ }^{44}$ Y. M. Ivanchen and L. A. Zilberma, Soviet Physics JETP-USSR 28, 1272 (1969).

${ }^{45}$ V. I. Tikhonov, Avtom. Telemekh. 20, 1188 (1959).

${ }^{46}$ P. S. Burada and G. Schmid, Phys. Rev. E 82, 051128 (2010).

${ }^{47}$ P. S. Burada, G. Schmid, and P. Hänggi, Phil. Trans. R. Soc. A 367, 3157 (2009).

${ }^{48}$ P. Hänggi and F. Marchesoni, Chaos 15, 026101 (2005).

${ }^{49}$ O. Pironneau, F. Hecht, and J. Morice, "freefem++,"

${ }^{50}$ S. Lifson and J. Jackson, J. Phys. Chem. 36, 2410 (1962).

${ }^{51}$ P. Kalinay and J. K. Percus, J. Chem. Phys. 122, 204701 (2005).

${ }^{52}$ P. Hänggi, T. J. Mroczkowski, F. Moss, and P. V. E. McClintock, Phys. Rev. A 32, 695 (1985)

${ }^{53}$ F. Moss, P. Hänggi, R. Mannella, and P. V. E. McClintock, Phys. Rev. A 33, 4459 (1986)

${ }^{54}$ R. Garcia, F. Moss, A. Nihongi, J. R. Strickler, S. Göller, U. Erdmann, L. Schimansky-Geier, and I. M. Sokolov, Math. Bios. 207, 165 (2007)

${ }^{55}$ L. Haeggqwist, L. Schimansky-Geier, I. M. Sokolov, and F. Moss, EPJ-ST 157, 33 (2008)

${ }^{56}$ J. Freund, L. Schimansky-Geier, B. Beisner, A. Neiman, D. F. Russel, T. Yakusheva, and F. Moss, J. Theo. Bio. 214, 71 (2002) 